\title{
An Eight-Week, Open-Label, Uncontrolled, Multicenter, Phase IV Study of Remission Rates in Outpatients and Inpatients with Major Depression Treated with Venlafaxine
}

Michel Dierick, MD, ${ }^{1}$ Andre De Nayer, MD, PhD, ${ }^{2}$ Marc Ansseau, MD, PhD, ${ }^{3}$ Hugo D'Haenen, MD, PhD, ${ }^{4}$ Paul Cosyns, MD, PhD, ${ }^{5}$ Ward Verbruggen, MD, ${ }^{6}$ Arlette Seghers, MD, PhD, ${ }^{7}$ Isidore Pelc, MD, $\mathrm{PhD}^{8}{ }^{8}$ Pierre Fossion, $\mathrm{MD}^{8}$ Grigori Stefos, MD, ${ }^{9}$ Joseph Peuskens, MD, PhD, ${ }^{10}$ Michel Malfroid, MD, ${ }^{11}$ Sophie Leyman, MD, ${ }^{12}$ and Annick Mignon, Pharm ${ }^{12}$

${ }^{1}$ Neuro-Psychiatrische Kliniek Sint Camillus, Gent, ${ }^{2}$ Hôpital Ste. Thérèse, Montignies-sur-Sambre, ${ }^{3}$ Centre Hospitalier Universitaire Sart Tilman, Liège, ${ }^{4}$ Academisch Ziekenhuis Vrije Universiteit Brussel, Brussels, ${ }^{5}$ Universitair Ziekenhuis Antwerpen, Edegem, ${ }^{6}$ Psychiatrisch Centrum Sint Amedeus, Mortsel, ${ }^{7}$ Cliniques Universitaires Saint Luc, Brussels, ${ }^{8}$ Centre Hospitalier Universitaire Brugmann, Brussels, ${ }^{9}$ Hôpital Universitaire St. Pierre, Brussels, ${ }^{10}$ Universitair Psychiatrisch Centrum Sint Jozef, Kortenberg, "Universitair Psychiatrisch Centrum Sint Kamillus, Bierbeek, and ${ }^{72}$ Wyeth Pharmaceuticals Belgium, Louvain-la-Neuve, Belgium

\section{ABSTRACT}

Background: Venlafaxine is a structurally novel antidepressant that is believed to potentiate monoamine activity in the central nervous system. In preclinical studies, venlafaxine was shown to inhibit the neuronal uptake of serotonin and norepinephrine and, to a lesser degree, dopamine reuptake, but was without effect on monoamine oxidase (MAO) activity. Clinical trial results from $\sim 3000$ patients suggest that venlafaxine is a safe and effective antidepressant with the potential to invoke an early onset of clinical activity.

Objective: The purpose of this 8-week, open-label, uncontrolled, multicenter, Phase IV study was to examine the extent of remission and symptom relief in outpatients and inpatients with major depressive disorder treated with venlafaxine.

Methods: This study was conducted at 12 centers across Belgiunn and Luxembourg. Consecutive, severely depressed inpatients and moderately depressed outpatients aged 18 to 70 years were eligible. Patients were adminis-

Part of this study was presented at the 11 th World Congress of Psychiatry, Berlin, Germany, August 6-11, 1999, and the 12th European College of Neuropsychopharmacology Congress, London, United Kingdom, September 21-25, 1999. 
tered open-label venlafaxine for 8 weeks. Dosing was initiated at venlafaxine $75 \mathrm{mg} / \mathrm{d}$ (37.5 mg BID), with dose adjustments made throughout the study, to a maximum daily dose of $375 \mathrm{mg}$ for inpatients and $225 \mathrm{mg}$ for outpatients. Results were measured using the Hamilton Depression (HAM-D) scale, the Montgomery-Asberg Depression Rating Scale (MADRS), and the Clinical Global Impression (CGI) scale.

Results: A total of 149 consecutive patients ( 84 females, 65 males; mean age, 46.5 years; 88 outpatients, 61 inpatients) were enrolled; the intent-to-treat (ITT) population comprised 144 patients (84 outpatients, 60 inpatients); 111 patients (64 outpatients, 47 inpatients) completed the study. At the week 8 visit, $71.3 \%$ of patients $(77 / 108)$ were considered to be responders according to the HAM-D scale; $73.8 \%$ (79/107) according to the MADRS; and 78.7\% (85/108) according to the CGI scale. A sustained response was achieved in $33.3 \%$ of the ITT population $(48 / 144)$, and at week $8,50.8 \%$ of outpatients $(32 / 63)$ and $37.8 \%$ of inpatients $(17 / 45)$ werc in remission according to the HAM-D scale. Venlafaxine was well tolerated at all doses, with the most frequently experienced adverse events (AEs) being nausea, sweating, and headache. Fewer inpatients than outpatients reported $\geq 1 \mathrm{AE}$ (57.4\% [35/61] and 73.9\% [65/88], respectively), despite receiving a higher maximum daily dose of venlafaxine.

Conclusion: The results of this study indicate that venlafaxine was a tolerable and effective antidepressant in both outpatients and inpatients, with a significant proportion of patients achieving remission.

Key words: venlafaxine, depression, remission, antidepressant. (Curr Ther Res Clin Exp. 2002;63:475-485)

\section{INTRODUCTION}

Venlafaxine is a structurally novel antidepressant that is believed to potentiate monoamine activity in the central nervous system. In a preclinical study, ${ }^{1}$ venlafaxine was shown to inhibit the neuronal uptake of serotonin and norepinephrine and, to a lesser degree, dopamine reuptake, but was without effect on monoamine oxidase (MAO) activity. The drug displays no affinity for rat brain muscarinic, cholinergic, histaninergic, or adrenergic receptors and, unlike other antidepressants, produces a rapid onset of noradrenergic subsensitivity. ${ }^{2}$

The human pharmacokinetic profile of venlafaxine was assessed in $>200$ patients in earlier Phase I and II studies. ${ }^{3,4}$ Orally administered venlafaxine is rapidly absorbed, with peak plasma venlafaxine concentrations attained within 2 hours $^{3}$ and plasma venlafaxine concentrations proportional to dose over the range of 25 to $75 \mathrm{mg}$ every 8 hours. Venlafaxine is extensively metabolized in the liver and eliminated predominantly by the kidney. ${ }^{4}$ The major urinary metabolite $O$-desmethylvenlafaxine also demonstrates serotonin and norepinephrine uptake inhibition in preclinical models, thus enhancing the pharmacologic efficacy of venlafaxine. ${ }^{1}$ 
Clinical trial results from $\sim 3000$ patients suggest that venlafaxine is a safe and effective antidepressant with the potential to invoke an early onset of clinical activity. In randomized, double-blind studies of venlafaxine with imipramine,$^{5-7}$ trazodonc, ${ }^{8}$ and placcbo ${ }^{9-11}$ in depressed patients, venlafaxine was more effective than placebo in relieving the symptoms of depression, and performed as well as, and in some cases better than, the comparative antidepressant drugs. In additional placebo-controlled clinical trials ${ }^{12}{ }^{14}$ performed in a generally equivalent manner, the most frequently reported adverse events (AEs) associated with venlafaxine administration were nausea, somnolence, dry mouth, and dizziness. These events were first observed after initial venlafaxine treatment and often subsided with continued drug administration. Dose-related increases in blood pressure ( $2 \mathrm{~mm} \mathrm{Hg}$ mean increase in supine diastolic blood pressure) and slight increases in heart rate also were reported.

This 8-week, open-label, uncontrolled, multicenter, Phase IV study, designed to represent typical clinical use of venlafaxine, assessed its ability to produce remission and symptom relief (defined as a score on the Hamilton Depression [HAM-D] scale $\leq 8$ ) in outpatients and inpatients with major depressive disorder.

\section{PATIENTS AND METHODS}

This study was conducted at 12 centers across Belgium and Luxembourg. All study procedures were conducted in accordance with the ethical principles detailed in the Declaration of Helsinki. Two hundred consecutive male and female outpatients and inpatients aged 18 to 70 years who had provided written informed consent were to be enrolled in this study. Inpatients were to have a minimum baseline score of 25 on the HAM-D scale and to be considered by the investigator to be severely depressed. Outpatients were to have a HAM-D score between 18 and 25 at baseline and to be considered moderately depressed. Women of childbearing potential were to have a negative pregnancy test result at baseline and were to use contraceptives throughout the study. Pregnant or breast-feeding women were not enrolled.

Also excluded were patients with concomitant psychiatric disease or personality disorder or known clinically significant abnormal laboratory test results. Patients were excluded if they had received any antidepressant, antipsychotic, or investigational drug within 7 days of baseline (unless the drug was permitted during the study period); MAO inhibitors within 14 days of baseline; fluoxetine within 21 days of baseline; or electroconvulsive therapy within 30 days of baseline. Medications prohibited during the study included psychotropic drugs, nonpsychotropic drugs with psychotropic effects (unless the dose had been stable for $\geq 1$ month), fluoxetine, MAO inhibitors, and antidepressants (except venlafaxine). Patients were, however, permitted to take $\leq 4$ $\mathrm{mg} / \mathrm{d}$ lorazepam or $\leq 2 \mathrm{mg} / \mathrm{d}$ lormetazepam, as well as other medications, to treat intercurrent medical conditions. Outpatients who were acutely suicidal were not enrolled. 
Patients were administered open-label venlafaxine for 8 weeks. Venlafaxine was taken orally, with meals. Outpatients initially received $75 \mathrm{mg}$ ( $37.5 \mathrm{mg}$ BID) venlafaxine for 2 weeks. Dose increases were allowed, in 75-mg increments at minimum intervals of 2 weeks, to a maximum daily dose of $225 \mathrm{mg}$ ( $75 \mathrm{mg}$ TID) to optimize the response. Inpatients received $75 \mathrm{mg} / \mathrm{d}(37.5 \mathrm{mg} \mathrm{BID})$ venlafaxine for 3 days, followed by $150 \mathrm{mg} / \mathrm{d}$ ( $75 \mathrm{mg}$ BID) for 6 days. Again, dose increases were allowed, in 75 -mg increments at minimum intervals of 3 days, to a maximum daily dose of $375 \mathrm{mg}$ (150 mg in the morning and evening and $75 \mathrm{mg}$ at noon). The decision to increase the dose of venlafaxine, or to reduce the dose in patients showing a sustained response, was based on tolerance and the clinical judgment of the investigator. However, outpatients unable to tolerate $\geq 75$ $\mathrm{mg} / \mathrm{d}$ and inpatients unable to tolerate $\geq 150 \mathrm{mg} / \mathrm{d}$ were withdrawn from the study.

At baseline (day 1), patients were assessed for eligibility, and a complete medical and psychiatric history was taken using patients' medical records and interviews. From baseline to week 8 , disease status was assessed using the HAM-D scale, the Montgomery-Asberg Depression Rating Scale (MADRS), and the Clinical Global Impression (CGI) scale. AEs and the use of concomitant medications also were recorded at these visits. Patients were withdrawn from the study if they experienced a marked increase in depression-especially the emergence of suicidal thoughts requiring precautionary action-that could not be controlled using study procedures.

\section{Statistical Analyses}

Statistical analyses were performed by Pharmaceutical Research Associates International (Charlottesville, Va) using the SAS software package version 6.08 (SAS Institute Inc, Cary, NC) and was based on pooled data from individual study centers. The intent-to-treat (ITT) data set comprised all patients who had received $\geq 1$ dose of venlafaxine and who had $\geq 1$ assessment during the treatment period, either while receiving therapy or within 3 days of the last dose. All analyses of efficacy and of vital signs for safety were performed using this population.

The primary efficacy variables were the week 8 on-therapy HAM-D and MADRS total scores. These scores were analyzed on the basis of all patients remaining in the study at each visit and on the basis of the last observation carried forward (LOCF). Vital-sign data were analyzed using summary statistics of changes from baseline and the incidence of vital signs outside predefined levels of concern.

Patients were considered to be responders if their total HAM-D score had decreased $\geq 50 \%$ from baseline, with an equivalent definition for MADRSdefined responders. They were considered overall responders if they were either a HAM-D- or MADRS-defined responder and they had a CGI score of 1 (very much improved) or 2 (much improved). Sustained response was defined as a response present at the end of the study that had lasted for $\geq 2$ weeks. Patients were considered to be in remission if they achieved a HAM-D score $\leq 8$. 
Data from all patients who received $\geq 1$ dose of study medication and who reported treatment-emergent AEs during the treatment period or within 7 days of the last dose were included in the analyses of study safety. Treatmentemergent AEs were defined as those not present, or present but less severe, at baseline.

\section{RESULTS}

A total of 149 consecutive patients ( 84 females, 65 males; mean age, 46.5 years; 88 outpatients, 61 inpatients) were enrolled. The ITT population comprised 144 patients (84 outpatients, 60 inpatients); 1 inpatient and 4 outpatients discontinued before postbaseline assessment and therefore were excluded because they failed to meet the requirements for eligibility for ITT. Before the first postbaseline assessment, 3 outpatients failed to return; 1 inpatient discontinued due to an $\mathrm{AE}$; and 1 outpatient discontinued due to lack of efficacy. All patients $(\mathrm{N}=149)$ were included in the tolerability analyses. Slightly more enrolled patients were women (55.7\% of outpatients [49/88], 57.4\% of inpatients [35/61] ), and the distribution of mean age, body weight, and height was similar between both patient groups. The mean age was 46.5 years (range, 20-76 years); mean body weight, $71.9 \mathrm{~kg}$ (range, 46-143 kg); and mean height, 168.8 $\mathrm{cm}$ (range, 145.5-193.0 cm).

\section{Baseline Assessments}

A summary of the depression status of all patients $(\mathrm{N}=149)$ at baseline is presented in Table I. At baseline, the mean ( \pm SD) HAM-D score for outpatients was $22.7 \pm 3.5$; for inpatients, $30.6 \pm 5.5$. The mean $( \pm \mathrm{SD})$ MADRS score for outpatients was $27.5 \pm 5.8$; for inpatients, $35.3 \pm 6.8$. According to the CGI scale, most patients were moderately, markedly, or severely ill. A total of $40.9 \%$ of all patients $(61 / 149 ; 54.5 \%$ of outpatients [48/88], $21.3 \%$ of inpatients [13/61]) were moderately ill; $37.6 \%$ of all patients $(56 / 149 ; 36.4 \%$ of outpatients [32/88], 39.3\% of inpatients [24/61]) were markedly ill; $16.1 \%$ of all patients $(24 / 149 ; 6.8 \%$ of outpatients [6/88], 29.5\% of inpatients [18/61]) were severely ill (Table I).

Overall, $91.9 \%$ of all patients $(137 / 149 ; 94.3 \%$ of outpatients [83/88], 88.5\% of inpatients [54/61]) had received prior antidepressant therapy, the most common type being selective serotonin reuptake inhibitors (SSRIs; $78.5 \%$ of all patients [117/149]).

\section{Efficacy Assessments}

Of the 111 patients who completed the study ( 64 outpatients, 47 inpatients), the numbers of patients assessed using the HAM-D, MADRS, and CGI scales were 108 ( 63 outpatients, 45 inpatients), 107 ( 63 outpatients, 44 inpatients), and 108 (63 outpatients, 45 inpatients), respectively.

Significant improvements in mean total HAM-D scores were observed $(P<0.001)$, with a mean reduction at week 8 of $15.4 \pm 8.1$ for all patients. Significant mean decreases in the scores of the following items of the HAM-D scale were seen at week 8 for all patients: anxiety/somatization $(3.0 \pm 2.5)$, 


\begin{tabular}{|c|c|c|}
\hline Parameter & $\begin{array}{l}\text { Inpatients } \\
(\mathrm{n}=61)\end{array}$ & $\begin{array}{l}\text { Outpatients } \\
(n=88)\end{array}$ \\
\hline \multicolumn{3}{|l|}{$\begin{array}{l}\text { Duration of current episode } \\
\text { of depression, no. (\%) }\end{array}$} \\
\hline $0-26 w k$ & $41(65.6)$ & $59(67.0)$ \\
\hline$>26-52 w k$ & $8(13.1)$ & $13(14.8)$ \\
\hline$>52$ wk & $6(9.8)$ & $15(17.0)$ \\
\hline Unknown & $6(9.8)$ & $1(1.1)$ \\
\hline $\begin{array}{l}\text { Mean HAM-D scale total score } \pm \\
\text { SD (range) }\end{array}$ & $30.6 \pm 5.5(22-47)$ & $22.7 \pm 3.5(18-36)$ \\
\hline $\begin{array}{l}\text { Mean MADRS total score } \pm \text { SD } \\
\text { (range) }\end{array}$ & $35.3 \pm 6.8(19-53)$ & $27.5 \pm 5.8(12-45)$ \\
\hline \multicolumn{3}{|l|}{ CGI scale asssessment, no. (\%) } \\
\hline Mildly ill & $0(0.0)$ & $2(2.3)$ \\
\hline Moderately ill & $13(21.3)$ & $48(54.5)$ \\
\hline Markedly ill & $24(39.3)$ & $32(36.4)$ \\
\hline Severely ill & $18(29.5)$ & $6(6.8)$ \\
\hline Extremely ill & $6(9.8)$ & $0(0.0)$ \\
\hline $\begin{array}{l}\text { Patients taking antidepressant } \\
\text { medication before study, no. (\%) }\end{array}$ & $54(88.5)$ & $83(94.3)$ \\
\hline
\end{tabular}

HAM-D = Hamilton Depression; MADRS = Montgomery-Asberg Depression Rating Scale; CGl = Clinical Global impression.

depressed mood $(2.0 \pm 1.2)$, sleep disturbance $(1.7 \pm 2.2)$, suicidal ideation $(1.2 \pm 0.9)$, retardation $(0.8 \pm 0.9)$, and agitation $(0.4 \pm 0.8)(P<0.001$ for all). The percentage of patients with a HAM-D suicidal ideation score of 0 (ie, absent) increased significantly $(P<0.001)$ from $20.3 \%$ of ITT patients $(29 / 143)$ at baseline to $80.6 \%(87 / 108)$ at week 8 , and the rate of HAM-D-defined responders was $71.3 \%(77 / 108 ; 71.4 \%$ of outpatients [45/63], 71.1\% of inpatients [32/45]) at week 8 and $56.3 \%(81 / 144 ; 54.8 \%$ of outpatients [ $46 / 84], 58.3 \%$ of inpatients [35/60]) at the final visit (LOCF).

Significant improvements in mean total MADRS scores were observed from baseline to week 8 in both patient groups (Figure $1 ; P<0.001$ ), with a mean decrease in MADRS score for all patients at week 8 of $19.7 \pm 10.9$. The mean decrease in total MADRS scores in both groups at the final visit (LOCF) was $16.1 \pm 12.4$. The percentage of all patients with an MADRS suicidal ideation score of 0 (ie, absent) increased significantly $(P<0.001)$, from $7.6 \%(11 / 144)$ at baseline to $69.2 \%(74 / 107)$ at week 8 , and the rate of MADRS-defined responders was $73.8 \%(79 / 107 ; 71.4 \%$ of outpatients [45/63], $77.3 \%$ of inpatients [34/44]) at week 8 and $59.7 \%(86 / 144 ; 56.0 \%$ of outpatients [47/84], $65.0 \%$ of inpatients [39/60] at the final visit (LOCF).

The rate of patients who were CGI-defined responders at week 8 was $78.7 \%$ $(85 / 108)$ compared with $18.8 \%(26 / 138)$ at week 1 . After 8 weeks of venlafaxine 


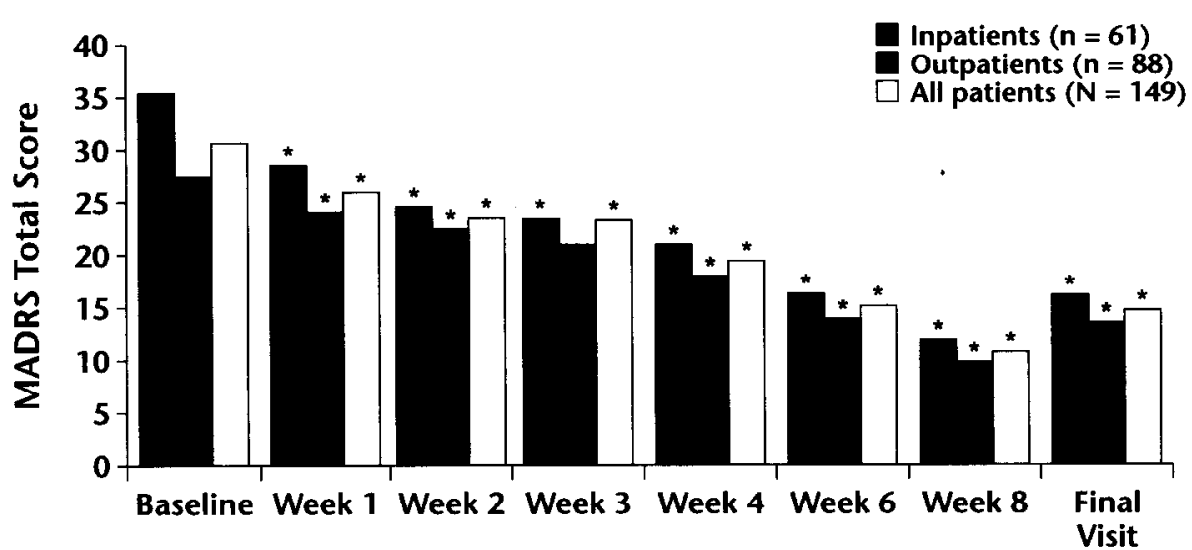

Figure 1. Mean Montgomery-Asberg Depression Rating Scale (MADRS) scores for all patients over the study period. ${ }^{*} P<0.001$ versus baseline.

administration, $69.4 \%$ of patients (75/108; $71.4 \%$ of outpatients [45/63], $66.7 \%$ of inpatients [30/45]) showed an overall response, with a sustained response observed in $33.3 \%$ of the ITT population (48/144; $33.3 \%$ of outpatients [28/84], $33.3 \%$ of inpatients [20/60]).

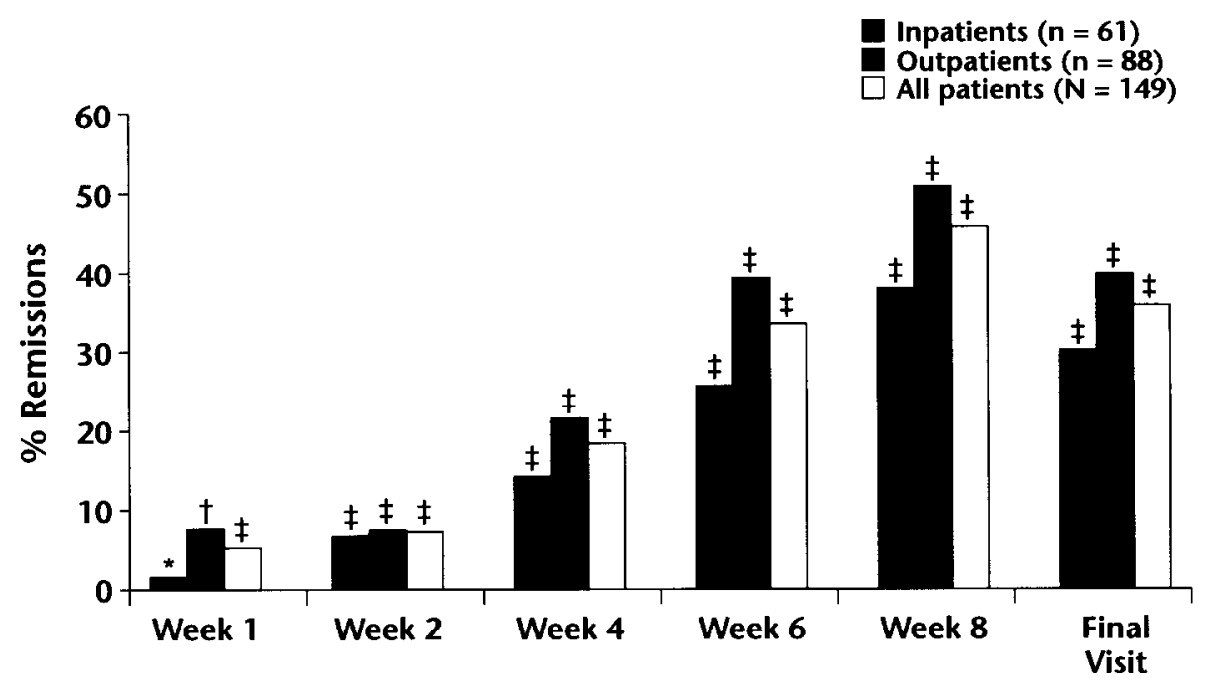

Figure 2. Rate of remissions as defined using the Hamilton Depression (HAM-D) scale (HAM-D score $\leq 8$ ). (The results for week 3 were flawed; data not shown.) $* P=0.001$ versus baseline; ${ }^{\dagger} P=0.003$ versus baseline; ${ }^{\ddagger} p<0.001$ versus baseline. 
By week $8,45.4 \%$ of patients $(49 / 108 ; 50.8 \%$ of outpatients [32/63], $37.8 \%$ of inpatients [17/45]) were in remission as defined by a HAM-D score $\leq 8$ (Figure 2 ). At the final on-therapy visit, the remission rate (LOCF) was $35.4 \%$ for the ITT population $(51 / 144 ; 39.3 \%$ of outpatients [33/84], $30.0 \%$ of inpatients [18/60]).

Information on venlafaxine dosing is shown in Table II. The median maximum daily dose levels of venlafaxine attained were $150 \mathrm{mg} / \mathrm{d}$ in the outpatient group and $225 \mathrm{mg}$ in the inpatient group. Among the outpatients, 65.9\% (58/88) attained a maximum daily dose compared with $72.1 \%(44 / 61)$ of inpatients. Twelve (19.7\%) inpatients had received a maximum daily dose of venlafaxine $>225 \mathrm{mg}$.

\section{Safety Assessments}

Overall, 25.5\% of all patients (38/149) withdrew from the study prematurely, with a slightly lower rate among inpatients (23.0\% [14/61]) than outpatients (27.3\% [24/88]). By week 1, 4.7\% of patients (7/149) had withdrawn (5 due to AEs, 1 due to failure to return, and 1 due to an unsatisfactory response). By week 4 , this rate had increased to $14.1 \%(21 / 149 ; 9$ of these patients withdrew between weeks 3 and 4). By week 8, 16 additional patients had withdrawn ( 8 patients between weeks 4 and 6 , and 8 patients between weeks 6 and 8 ), with $24.8 \%$ of patients (37/149) withdrawn overall; 1 patient withdrew after week 8 . The most common reasons for discontinuation were the occurrence of an $\mathrm{AE}(10.1 \%$ [15/149]) and unsatisfactory response $(8.7 \%$ [13/149]).

Venlafaxine was well tolerated at all doses, and the most frequently reported treatment-emergent AEs in all patients were nausea (24.2\% [36/149]), sweating (12.1\% [18/149]), and headache (10.1\% [15/149]). Although inpatients received, on average, a higher maximum daily dose of venlafaxine than did outpatients, fewer inpatients reported treatment-emergent AEs (57.4\% of inpatients [35/61], $73.9 \%$ of outpatients [65/88]), with markedly lower occurrence rates of nausea, sweating, and headache. Most of these AEs were considered to be treatment related.

No clinically significant changes in systolic or diastolic blood pressure values were observed during the study. However, a substantial mean increase in

Table II. Venlafaxine dosing information for all study patients $(N=149)$.

\begin{tabular}{lcc}
\hline Parameter & Inpatients $(\mathrm{n}=61)$ & Outpatients $(\mathrm{n}=88)$ \\
\hline Mean dose $\pm \mathrm{SD}, \mathrm{mg} / \mathrm{d}$ & $156.5 \pm 99.1$ & $113.7 \pm 33.8$ \\
Maximum daily dose attained, & & \\
no. $(\%)$ & & \\
$75 \mathrm{mg}$ & $5(8.2)$ & $28(31.8)$ \\
$112.5 \mathrm{mg}$ & $0(0.0)$ & $1(1.1)$ \\
$150 \mathrm{mg}$ & $17(27.9)$ & $26(29.5)$ \\
$225 \mathrm{mg}$ & $27(44.3)$ & $32(36.4)$ \\
$>225 \mathrm{mg}$ & $12(19.7)$ & $1(1.1)$ \\
\hline
\end{tabular}


heart rate of $3.3 \pm 11.9$ beats/min was observed for all patients at week 8 . In total, $4.7 \%$ of patients (7/149) experienced increases or decreases of $>7.0 \%$ in body weight in their baseline measurements at week 8 .

\section{DISCUSSION}

The antidepressant efficacy of venlafaxine has been proved in both placebocontrolled and active comparator-controlled studies. ${ }^{5-11}$ The purpose of the present study was not only to confirm these findings but also to observe how the drug performed in clinical practice. Consecutive patients were enrolled in this Phase IV study to examine the use of venlafaxine within a representative population of depressed patients, as opposed to the highly selected patient populations examined in Phase II and III clinical trials.

This study was run in psychiatric inpatient and outpatient settings; consequently, most patients had experienced prior depressive episodes and had been previously treated for depression. No comparator drug was used, and placebo was considered unethical for use in severely depressed patients. The use of open-label venlafaxine, combined with each investigator's option to adjust dose depending on the patient's clinical response and tolerance, introduced the possibility of investigator bias and interinvestigator variability; however, the results obtained support those previously observed in controlled clinical trials of venlafaxine.

Most earlier studies have determined the efficacy of venlafaxine in terms of response, as defined by decreases in total HAM-D and MADRS scores $\geq 50 \%$ from baseline. However, the main focus of the present study was patient remission, defined as an end-of-study HAM-D score $\leq 8$. Indeed, of those patients who remained on venlafaxine therapy for 8 weeks, $50.8 \%(32 / 63)$ of outpatients and $37.8 \%(17 / 45)$ of inpatients achieved remission-a state that can be defined as virtually asymptomatic-despite moderate or severe depression scores at baseline.

Although no comparator drug was used, this high level of remission confirms rates observed in previous studies of venlafaxine. In outpatients, Ballus et $\mathrm{al}^{15}$ compared venlafaxine to paroxetine in patients with major depression or dysthymia. At week 12 of treatment, significantly more patients in the venlafaxinetreated group than in the paroxetine-treated group had a HAM-D remission score $\leq 8(59 \%$ vs $31 \%$ of patients; $P=0.011)$. Results from a comparative study published by Mehtonen et $\mathrm{al}^{16}$ also showed a statistically significantly higher $(P<0.05)$ remission rate (HAM-D score $<10)$ at week 8 for patients treated with venlafaxine ( $68 \%$ of patients) versus patients treated with sertraline $(45 \%$ of patients). In a 6-week study in inpatients, Tzanakaki et al ${ }^{17}$ found higher remission rates (HAM-D score $<7$ ) with venlafaxine ( $41 \%$ of patients) compared with fluoxetine ( $36 \%$ of patients). Nierenberg et $\mathrm{al}^{18}$ assessed the use of venlafaxine in consecutive moderately depressed outpatients and inpatients who had failed to respond to previous antidepressant therapies. After 12 weeks of treatment, $22 \%$ of patients remaining in the study were in remission. A meta-analysis of the 
pooled results from 8 studies in which venlafaxine was compared with SSRIs showed more rapid and higher remission rates (HAM-D score $\leq 7$ ) with venlafaxine compared with the SSRIs analyzed. ${ }^{19}$

In the present study, significant improvements in mean total HAM-D and MADRS scores were seen for both patient groups $(P<0.001$ for all), with a significantly greater percentage of patients having reduced HAM-D-defined suicidal ideation and retardation scores and MADRS total scores by week 8 compared with baseline $(P<0.001$ for all). Because these 2 symptoms of depression often result in patient hospitalization, the significant improvements observed are of social and economic importance. In addition, improvements in mean individual HAM-D scores for anxiety/somatization, sleep disturbance, depressed mood, and agitation were noted in both outpatients and inpatients at week 8.

Venlafaxine was well tolerated, particularly in inpatients, who received higher maximum daily doses than did outpatients but reported fewer AEs. The types of AEs experienced were as expected for patients receiving venlafaxine, with nausea, sweating, and headache reported most frequently. Overall, $25.5 \%$ $(38 / 149)$ of patients withdrew from the study-10.1\% due to AEs and $8.7 \%$ due to lack of efficacy. Compared with other studies in patients with similar levels of depression, ${ }^{20}$ this rate of withdrawal was low. No anticipated significant abnormalities in blood pressure occurred during the course of the study, further demonstrating the tolerability of venlafaxine.

\section{CONCLUSION}

The results of this study suggest that venlafaxine was a tolerable and effective antidepressant, which was able to provide remission and symptom relief in a significant proportion of moderately or severely depressed inpatients and outpatients in a natural setting.

\section{ACKNOWLEDGMENTS}

Funding for this study was provided by Wyeth-Ayerst Global Medical Affairs (Collegeville, Pennsylvania). The manuscript was prepared by Quintiles Ltd., Bracknell, United Kingdom.

\section{REFERENCES}

1. Muth EA, Haskins JT, Moyer JA, et al. Antidepressant biochemical profile of the novel bicyclic compound $\mathrm{Wy}-45,030$, an ethyl cyclohexanol derivative. Biochem Pharmacol. 1986;35:4493-4497.

2. Muth EA, Moyer JA, Haskins JT, et al. Biochemical, neurophysiological, and behavioral effects of $\mathrm{Wy}-45,233$ and other identified metabolites of the antidepressant venlafaxine. Drug Dev Res. 1991;23:191-199.

3. Fabre LF, Putman HP. An ascending single-dose tolerance study of Wy-45,030, a bicyclic antidepressant, in healthy men. Curr Ther Res Clin Exp. 1987;42:901-909.

4. Howell SR, Husbands GE, Scatina JA, Sisenwine SF. Metabolic disposition of ${ }^{14} \mathrm{C}$-venlafaxine in mouse, rat, dog, rhesus monkey and man. Xenobiotica. 1993;23: 349-359. 
5. Schweizer E, Feighner J, Mandos LA, Rickels K. Comparison of venlafaxine and imipramine in the acute treatment of major depression in outpatients. J Clin Psychiatry. 1994;55:104-108.

6. Ferguson J, Khan A, Kucharik R, et al. A placebo-controlled comparative study of the effects on blood pressure and antldepressant efficacy of venlafaxine and inipraninte [poster]. Neuropsychopharmacology. 1994;10(Suppl):165S.

7. Benkert $\mathrm{O}$, Gründer G, Wetzel H, Hackett D. A randomized, double-blind comparison of a rapidly escalating dose of venlafaxine and imipramine in inpatients with major depression and melancholia. $J$ Psychiatr Res. 1996;30:441-451.

8. Cunningham LA, Borison RL, Carman JS, et al. A comparison of venlafaxine, trazodone, and placebo in major depression. J Clin Psychopharmacol. 1994;14:99-106.

9. Guelfi JD, White C, Hackett D, et al. Effectiveness of venlafaxine in patients hospitalized for major depression and melancholia. J Clin Psychiatry. 1995;56:450-458.

10. Khan A, Fabre LF, Rudolph R. Venlafaxine in depressed outpatients. Psychopharmacol Bull. 1991;27:141-144.

11. Schweizer E, Weise C, Clary C, et al. Placebo-controlled trial of venlafaxine for the treatment of major depression. I Clin Psychopharmacol. 1991;11:233-236.

12. Rudolph RL, Fabre LF, Feighner JP, et al. A randomized, placebo-controlled, doseresponse trial of venlafaxine hydrochloride in the treatment of major depression. J Clin Psychiatry. 1998;59:116-122.

13. Rudolph RL, Feiger AD. A double-blind, randomized, placebo-controlled trial of oncedaily venlafaxine extended release (XR) and fluoxetine for the treatment of depression. $J$ Affect Disord. 1999;56:171-181.

14. Silverstone PH, Ravindran A, for the Venlafaxine XR 360 Study Group. Once-daily venlafaxine extended release (XR) compared with fluoxetine in outpatients with depression and anxiety. J Clin Psychiatry. 1999;60:22-28.

15. Ballus C, Quiros G, De Flores T, et al. The efficacy and tolerability of venlafaxine and paroxetine in outpatients with depressive disorder or dysthymia. Int Clin Psychopharmacol. 2000;15:43-48.

16. Mehtonen OP, Sogaard J, Roponen P, Behnke K, for the Venlafaxine 631 Study Group. Randomized, double-blind comparison of venlafaxine and sertraline in outpatients with major depressive disorder. J Clin Psychiatry. 2000;61:95-100.

17. Tzanakaki M, Guazzelli M, Nimatoudis I, et al. Increased remission rates with venlafaxine compared with fluoxetine in hospitalized patients with major depression and melancholia. Int Clin Psychopharmacol. 2000;15:29-34.

18. Nierenberg AA, Feighner JP, Rudolph R, et al. Venlafaxine for treatment-resistant unipolar depression. J Clin Psychopharmacol. 1994;14:419-423.

19. Thase ME, Entsuah AR, Rudolph RL. Remission rates during treatment with venlafaxine or selective serotonin reuptake inhibitors. Br J Psychiatry. 2001;178:234-241.

20. Einarson TR, Addis A, Mittmann N, Iskedjian M. Meta-analysis of venlafaxine, SSRIs and TCAs in the treatment of major depressive disorder. Can J Clin Pharmacol. $1998 ; 5: 205-216$.

\section{Address correspondence to:}

Annick Mignon, Pharm

Wyeth Pharmaceuticals Belgium

Rue du bosquet 15

1348 Louvain-la-Neuve

Belgium 